
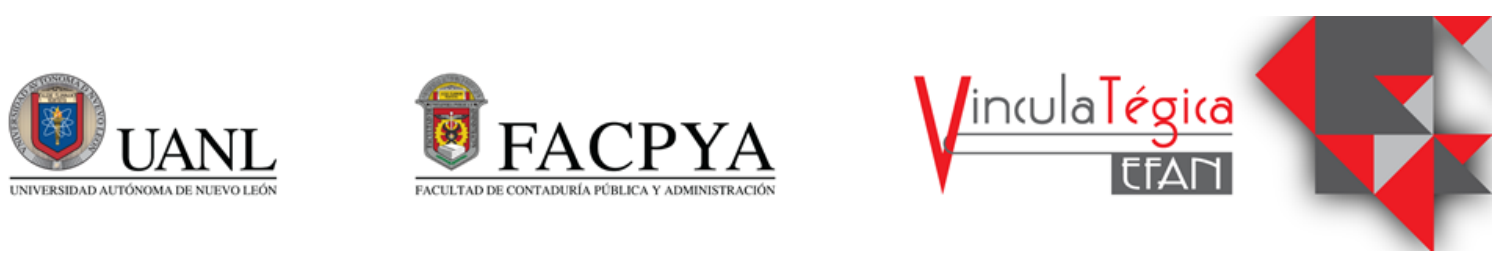

\title{
Diseño de instrumento que permite analizar el impacto de las variables de salarios y liderazgo en la rotación laboral de la empresa TDV dedicada a la elaboración de tarimas en Mexicali, Baja California, México
}

\author{
Refugio del Pilar García Sandoval ${ }^{1}$, Luis Ramón Moreno Moreno ${ }^{2}$ y Gloria Muñoz Del \\ $\mathrm{Real}^{3}$ \\ ${ }^{1}$ Universidad Autónoma de Baja California, Facultad de Ciencias Administrativas Mexicali, Baja California, \\ México,137925@uabc.edu.mx, Calzada de los presidentes y Eje central, Río Nuevo, 21120 Mexicali, B.C., \\ (+52) 6862478162 \\ ${ }^{2}$ Universidad Autónoma de Baja California, Facultad de Ciencias Administrativas Mexicali, Baja California, \\ México,lmoreno@uabc.edu.mx, Calzada de los presidentes y Eje central, Río Nuevo, 21120 Mexicali, B.C., \\ (+52) 6861741165 \\ ${ }^{3}$ Universidad Autónoma de Baja California, Facultad de Ciencias Administrativas Mexicali, Baja California, \\ México, gloria.munoz@uabc.edu.mx, Calzada de los presidentes y Eje central, Río Nuevo, 21120 Mexicali, \\ B.C., (+52) 6865823334
}

\author{
Información del artículo revisado por pares \\ Fecha de aceptación: junio-2021 \\ Fecha de publicación en línea: diciembre-2021 \\ DOI: https://doi.org/10.29105/vtga7.1-115
}

Resumen En la actualidad debido a la globalización y constante cambio que se presentan en las organizaciones de todos tamaños, es de extrema importancia contar con elementos que les permitan la toma de decisiones correctas y en tiempo actual, todo esto enfocado a la reducción de costos, efectividad operativa y aprovechamiento del recurso más importante de toda organización el talento de sus colaboradores, ya que la correcta gestión del talento sigue tomando una mayor importancia a diario, en las condiciones actuales del mundo empresarial. El objetivo de este trabajo es el diseño de un instrumento que permita medir el impacto que tienen las variables de sueldos y liderazgo sobre la rotación laboral presentada en la empresa TDV ubicada en Mexicali, B.C. y dedicada a la elaboración de tarimas. Después de una prueba piloto a un pequeño grupo de la muestra seleccionada, los resultados se analizaron por medio del sistema estadístico IBM SPSS, mostrando que todas las pruebas aplicadas a las dimensiones analizadas en el instrumento se encuentran dentro de los parámetros adecuados, concluyendo así, que el instrumento es adecuado para medir la influencia de las variables independientes sobre la variable dependiente.

Palabras clave: rotación laboral, salarios, liderazgo, diseño de instrumentos, pruebas validación.

\begin{abstract}
Currently due to globalization and constant change that occur in organizations of all sizes, it is extremely important to have elements that allow them to make correct decisions and in current time, all this focused on reducing costs, effectiveness operational and use of the most important resource of any organization, the talent of its collaborators, since the correct management of talent continues to take on greater importance on a daily basis, in the current conditions of the business world. The object of this study, it's the design of an instrument that allows to measure the impact that has the variables of salary and leadership over the job rotation happening in the company TDV, located in Mexicali, Baja California dedicated to the production of pallets. After a pilot test to a small group of the selected
\end{abstract}


sample, the results were analyzed by the statistics system IBM SPSS, showing that all the test applied to the dimensions analyzed in the instrument are on the right parameters, concluding that the instrument it's suitable to measure the influence of the independent variable over the depending variable.

\section{INTRODUCCIÓN}

\subsection{Planteamiento del problema}

Uno de los principales problemas que las empresas deben resolver a diario es la rotación laboral, que se define como el flujo constante de salida y entrada de su fuerza laboral (Chiavenato, 2009). Dicho problema impacta a la empresa de manera económica, debido a los costos de reclutamiento, selección, contratación, capacitación, entrenamiento, y la baja en productividad por la curva de aprendizaje de los nuevos elementos; por lo cual el ambiente organizacional resulta afectado debido al desequilibrio que genera una vacante en la empresa (Chiavenato, 2011).

En el presente trabajo se aborda el caso de la empresa TDV, dedicada a la venta de tarimas y estructuras para empaque todo elaborado de madera, ubicada en la ciudad de Mexicali, Baja California, México. En 2015 la empresa inició una reestructuración en su administración, que la llevó a la reforma en sus métodos de trabajo, de inversión, su cartera de clientes y de su planta de empleados, la cual contaba con 108 en ese año, modificándose a partir de 2016 a un requerimiento mínimo para laborar de 180 empleados. Haciendo el análisis de los datos del porcentaje de rotación de la empresa para el periodo 2015-2019 se observa que dicho indicador ha fluctuado entre un $20 \%$ y $46 \%$ anual, situación que desemboca en un alto costo económico para la empresa debido al tiempo de entrenamiento invertido, las horas extras que se generan debido a la baja productividad por la curva de aprendizaje del grueso de la población laboral de "nuevo ingreso", ya que las personas con menor antigüedad son las que continuamente entran y salen de la empresa (Herrera Arau, 2018).
Keywords: job rotation, salaries, leadership, instrument design, validitation tests.

JEL: J63, M12, M21, M51.

Basados en esta problemática que se genera en la empresa analizada, se busca diseñar y validar un instrumento que permita medir el impacto que tienen los factores de sueldo y liderazgo en la rotación de personal que se presenta en el área productiva. Ya contando con un instrumento que permita evaluar estos factores y sus relaciones, se enfocará a la aplicación de dicho instrumento en la población elegida para poder obtener resultados fiables que permitan a la empresa el diseño de estrategias adecuadas y canalizadas a la disminución de los índices de rotación laboral que se manejan actualmente.

\subsection{Pregunta de investigación}

¿Es posible que por medio del diseño y validación de un instrumento se pueda medir la causa principal de la constante rotación laboral en la empresa TDV?

\subsection{Hipótesis}

La aplicación de un instrumento validado permitirá identificar las causas y el impacto que éstas tienen respecto a la rotación de personal de la empresa TDV.

\subsection{Objetivo}

Diseñar y validar un instrumento que permita identificar el impacto de las variables de sueldos y liderazgo en la rotación laboral que afecta a la empresa TDV.

\section{MARCO TEÓRICO}

En este apartado se abordan las teorías utilizadas para la operacionalización de las variables, finalizando con definiciones de autores sobre las pruebas estadísticas aplicadas y los valores que se consideran adecuados al validar un instrumento de investigación.

\subsection{Rotación Laboral}

El recurso humano es el elemento más importante para que las organizaciones 
funcionen, realicen sus actividades día a día, generen ganancias, crezcan y desarrollen características que le permiten ser parte del mercado en que se desenvuelven y sean competitivas, debido a los conocimientos y habilidades con las que cada uno de los colaboradores cuenta y que aportan a las empresas de las que forman parte.

Debido a las características tan personales e individuales que tiene el factor humano es importante que la empresa ponga especial atención en atender las necesidades de su talento con el fin de mantenerlo dentro de la empresa y evitar que el personal con las habilidades necesarias salga de la empresa por diversos factores, disminuyendo así la rotación de personal que afecta a la organización debido la decisión de estos de separarse de la empresa.

Como define Santibañez, (2001, citado en Herrera, 2018):

... la rotación en el empleo es la salida voluntaria de un trabajador de la empresa en la que labora, esto ocasionado por diversos factores como la baja remuneración, la monotonía del empleo, la falta de reconocimiento a su labor, el clima laboral, el acoso, el estrés laboral, la excesiva demanda de trabajo y poca mano de obra, lo que conlleva a que un solo empleado termine realizando las actividades de dos o tres compañeros; ocasionando inconformidad. (p. 26)

Se puede observar que en ocasiones el trabajador decide, por razones personales o profesionales, separarse de la organización y en otras ocasiones la empresa busca la separación del trabajador debido a diversas razones como una inadecuada selección o reducción de fuerza laboral, sin embargo, ambos tipos de salida de la organización afectan los porcentajes de rotación laboral. (Chiavenato, 2009)

$\mathrm{Si}$ la empresa es quien se encuentra provocando la rotación debido a la búsqueda en el mercado laboral de mejores elementos, la rotación se encuentra en control de esta. Pero si el recurso humano es quien está tomando la decisión de separarse del centro de trabajo, es necesario que la entidad ponga atención a la rotación y sobre todo a identificar la causa que está provocando estas salidas y buscar evitar o disminuir a niveles deseables la perdida de personal importante para las operaciones diarias. (Chiavenato, 2011)

La rotación laboral no se debe estudiar como una causa sino como un efecto, conforme a Chiavenato (2011) se observan los siguientes factores que inciden en mayor o menor grado en la rotación laboral de las organizaciones: a) Externos a la organización: oferta y demanda en el mercado de los recursos humanos, situación económica del país o región, ofertas de trabajo que se dan en el mercado laboral; b) Internos a la organización: política de sueldos que se maneja en la organización, prestaciones adicionales que se ofrecen, tipos de supervisión, oportunidad de desarrollo laboral, entorno físico en que se desarrollan las actividades, cultura organizacional, evaluaciones de desempeño de los colaboradores, programas de capacitación, técnicas de reclutamiento y selección del recurso humano.

Es importante mencionar algunos efectos negativos de este fenómeno laboral que impactan a la empresa de manera directa; que son los costos en los que se incurren por concepto de reclutamiento, selección, contratación, capacitación, entrenamiento, además de la baja en productividad por curva de aprendizaje de los nuevos elementos; que por ende, el ambiente organizacional resulta afectado por el desequilibrio que genera una vacante en la empresa (Chiavenato, 2011). Debido a estos costos se afecta la efectividad de la empresa, por lo cual los administradores a cargo de estas deben prestar atención a la calidad de la vida laboral de sus colaboradores (Aldape et al., 2011).

\subsection{Sueldos.}

De acuerdo con el modelo de Jerarquías de Maslow (Chiavenato, 2011), se infiere que el principal motivo por el cual toda persona busca un trabajo es satisfacer sus necesidades primarias y al tener cubiertas éstas se buscará la satisfacción de las necesidades secundarias.

Por lo anteriormente expuesto se puede inferir que, si el salario de los 
colaboradores no alcanza a cubrir las necesidades primarias, se generará una insatisfacción que derivará en la búsqueda de un nuevo empleo. En este caso la organización debe buscar siempre en primer lugar que sus remuneraciones logren cubrir las necesidades básicas de sus colaboradores, partiendo de ahí se podrán orientar a cubrir otras necesidades que vayan enfocadas a la satisfacción de su personal en sus puestos de trabajo; el armonizar este principio fundamental con los objetivos que la empresa desea alcanzar, es necesario, ya que se reflejara el bienestar de la organización por medio del bienestar de sus colaboradores.

Werther y Davis, (2000, citado en Flores et al., 2008) sostienen que: "Un elemento esencial para mantener y motivar la fuerza de trabajo es la compensación adecuada. Los empleados deben recibir sueldos y salarios justos a cambio de una contribución productiva." (pp. 70-71) En este punto es importante indicar que una labor de los encargados del área de recursos humanos es mantener, junto con los administradores y tomadores de decisiones estos estándares en los sueldos y salarios al momento de revisar las políticas que los determinan, con la finalidad de disminuir el impacto de esta variable en la rotación de sus colaboradores.

En México el marco Legal que protege el salario que debe pagarse a los trabajadores es la Ley Federal del trabajo, en su artículo 82 define al salario como “... la retribución que debe pagar el patrón al trabajador por su trabajo" y en su artículo 85 indica que "el salario debe ser remunerador y nunca menor al fijado como mínimo de acuerdo con las disposiciones de ésta Ley" (Ley Federal del Trabajo, 2019, p. 24). Por lo que en México el punto de partida para el pago a los trabajadores se basa en este precepto legal y el pago por encima de los importes señalados por la Ley son un punto de competitividad para que sus colaboradores permanezcan con ellos.

Las organizaciones pueden buscar diferentes formas de reconocer el trabajo de sus colaboradores no solamente mediante el pago de salarios, sino por medio de las compensaciones, otorgándose de manera clara, por destacarse en las labores asignadas, o por ciertas conductas que le den un extra a la organización y la lleven a lograr sus objetivos; regularmente las empresas buscan mantener su competitividad salarial por medio de estos pagos adicionales como los son; bonos de puntualidad, asistencia, productividad, despensa, gatos médicos, becas escolares (Barón Ramírez, 2020).

Conforme a los resultados obtenidos en un trabaja realizado en una empresa maquiladora en México, elaborado por Carillo et al. (2001, citado en Domínguez Olaya, 2015), se muestra que dentro de las causas de porque los trabajadores en este tipo de empresas dejan su puesto de trabajo, se debe a que ofrecen salario y prestaciones bajas, lo cual reafirma la incidencia de esta variable en la rotación laboral.

En el trabajo de Aranibar (2018) se concluye la importancia de que la empresa haga sentir parte de la organización a los empleados por medio de salarios y prestaciones que los motiven y que al mismo tiempo desarrollen una mayor productividad en la empresa, estudio llevado a cabo en un grupo de maquiladoras de la ciudad de Ensenada Baja California, México, enfocado al personal productivo de las mismas.

En el estudio efectuado por Patista et al. (2019) sobre los factores que inciden en la rotación laboral, se enfoca a los empresarios para saber su opinión sobre los factores principales que ellos perciben y que afectan los índices de rotación en sus empresas, indicándose como uno de los principales las remuneraciones que se otorgan a sus colaboradores entre otros factores que no se analizarán en ese trabajo.

Sin embargo, una investigación realizada en una empresa maquiladora ubicada en la ciudad de Ensenada, Baja California, México, enfocándose al personal de producción, proporcionó como conclusión que aún cuando las prestaciones tienen una relación directa, no es un medio para contrarrestar la salida de personal, por lo que se deben analizar otros factores adicionales a las prestaciones. (Barón Ramírez, 2020)

\subsection{Liderazgo}

Un tema determinante para disminuir la rotación laboral al momento de revisar 
investigaciones previas es la satisfacción laboral que se relaciona directamente con el fenómeno de rotación laboral (Llanes Barreras, 2019). Por lo que es necesario conocer cómo se define la satisfacción laboral y qué la determina:

La satisfacción laboral es una actitud que los individuos tienen acerca de sus empleos; resulta de las percepciones de sus puestos y se basa en factores del ambiente laboral, como el estilo del supervisor, las políticas y procedimientos, la afiliación al grupo de trabajo, las condiciones de trabajo y las prestaciones. (Gibson et al., 2011, p. 102)

El liderazgo no sólo es una manera de influir en los demás de acuerdo con Newstrom (2011), es también una forma de apoyo para que se trabaje enfocados en el logro objetivos de manera entusiasta, en esta definición se comienza a entender la importancia de este concepto en la adaptación de los colaboradores al grupo y su identificación con la empresa, lo que incide en su decisión de permanencia.

Las empresas deben buscar que sus líderes no sólo ejerzan la autoridad por la posición jerárquica que se les designa de manera formal, sino que sean capaces de definir metas y comunicarlas adecuadamente a sus colaboradores con el fin de lograr los objetivos de las organizaciones para las cuales trabajan; por lo que un líder que no sabe comunicarse adecuadamente con sus subordinados genera estrés e insatisfacción laboral lo que lleva en última instancia, al aumento en la rotación laboral de su área o departamento. (Robbins, 2009)

Robbins (2009) habla de diversas teorías del liderazgo y sus enfoques; a) Teorías basadas en las características: se centran en las cualidades y características personales, describiendo a los líderes con términos como entusiastas, valientes carismáticos, sin embargo, que una persona posea ciertas características no determina el éxito para lograr los objetivos del grupo; b) Teorías basadas en el comportamiento: enfocándose a los comportamientos determinados de los líderes eficaces en tiempos difíciles, encontrándose que si fuera de esta manera sería posible la capacitación de líderes y se tendría un sinnúmero de líderes eficaces; c) Teorías basadas en la contingencia: observándose que ciertos comportamientos y características funcionaron en ciertas situaciones, pero no a largo plazo, el liderazgo que pudo funcionar en una empresa en una época determinada puede ser eficiente si cambia alguna de las condiciones; d) Teoría del intercambio líder-miembro: en esta teoría se plantea que el líder desarrolla una relación especial con ciertos miembros del grupo, desarrollándose un grupo interno con privilegios especiales por parte del líder y un grupo externo sólo desarrollándose una autoridad formal; e) Teoría de la decisión (Modelo de la participación del líder de Vroom y Yetton): en esta teoría la forma en la que el líder toma las decisiones es tan importante como la decisión misma; el comportamiento del líder debe ajustarse a la estructura de la tarea.

La importancia de conocer estas teorías es el reconocimiento y ajuste del tipo de liderazgo al tipo de organización y las tareas que se requieren para lograr los objetivos planteados. "la cultura es una [ sic] aspecto que está directamente relacionado con el liderazgo, ya que los tipos de liderazgo y/o teorías influyen más dependiendo de la cultura, tal como lo confirma Geert Hofstede en su teoría de las dimensiones culturales." (Cifuentes Cadena, 2017, p. 17)

En referencia al párrafo anterior, Kras (1989 citado por Vázquez, 2006) con respecto al liderazgo determinado por la cultura dice:

... en México se puede encontrar tanto un liderazgo tradicional como un estilo moderno. En una organización tradicional, los mexicanos consecutivamente transfieren ciertas dinámicas familiares al lugar de trabajo, el líder o dueño de estas compañías es visto como una figura paternal y frecuentemente usa un estilo de dirección autocrático. (p. 3)

El primer contacto de todo trabajador es su líder y si éste no tiene ni el perfil, ni la preparación adecuada, desmotivará al subordinado y habrá un aumento en la rotación del personal que depende de ese líder en 
particular; si la empresa sólo busca líderes que se comprometan con los objetivos productivos, sin comprometerse con el personal a su cargo, los objetivos de la empresa y el personal no estarán alineados, razón por la cual el papel de líder se vuelve importante ya que es el encargado de alinear los objetivos y motivar al personal que se encuentra a su cargo a cumplirlos de la mejor manera y sintiéndose parte importante de cada logro de la organización. "Los líderes ayudan al grupo a lograr sus objetivos aprovechando al máximo sus capacidades...a modo de facilitarle el progreso e inspirarle para lograr las metas organizacionales." (Koontz \& Weihrich, 2013, p. 302). Adicionalmente, se demostró en una en una investigación de Steers y Porter (1983) como se citó en Benavides et al. "la influencia de la satisfacción en el trabajo, la retribución monetaria y el estilo del supervisor en la rotación." (s/f, p. 5)

En el estudio efectuado en una empresa en Mexicali, Baja California, México, se llegó a la conclusión que la interacción entre los líderes de producción y sus subordinados es una determinante para lograr disminuir la rotación laboral, así como lo es la capacitación de los supervisores, enfocándose al manejo adecuado del personal y la resolución de conflictos, con el fin de que desarrollen confianza y una adecuada comunicación con el personal a su cargo, ya que de ellos depende que el empleado se sienta apoyado y parte importante de la empresa, así como de las decisiones que se toman dentro de la misma. (Ramos García, 2018).

En los hallazgos del estudio realizado por García et al. (2020) se resalta que para lograr mejorar y generar un alto sentido de pertenencia con la organización, se deben fortalecer el ambiente en que se labora por medio de establecer mejores relaciones entre jefes y subordinados.

Cómo se puede observar de acuerdo con los estudios más recientes sobre rotación laboral, las organizaciones deben enfocarse aún más en la persona, y no tanto en el trabajo que va a desempeñar, el hacer sentir importantes a cada uno de los colaboradores, haciéndolos participar en las decisiones que se toman a diario para el desarrollo de sus labores, fomenta la identificación con los valores, propósitos y objetivos de la organización. El nombre de recurso humano va quedando obsoleto en la actualidad ya que la empresa es un ser interrelacionado y unido por el talento de cada individuo que la conforma.

\subsection{Fiabilidad y Validez del instrumento}

Contar con un instrumento fiable que indique que se puede aplicar en diferente tiempo, bajo las mismas condiciones y arrojará resultados consistentes, midiendo aquel fenómeno para lo cual fue diseñado, Tarazona la define como "la consistencia de la medida del rasgo o característica para el cual fue diseñado, teniendo en cuenta los posibles errores que pueden alterar la medición." (2020, p. 183).

Para efecto de medir la fiabilidad del instrumento se utilizó el Alpha de Cronbach, debido que se tiene un instrumento con respuestas tipo Likert, como lo menciona Canu "el coeficiente Alpha de Cronbach es una generalización de la fórmula 20 de Kuder y Richardson para ítems con evaluación numérica no dicotómica" (2017, p. 3). Los valores que se buscan al aplicar esta prueba pueden variar de 0.90 indicado un grado de fiabilidad alto, aceptable si se arroja un valor de 0.75 y aquellos valores mayores a 0.50 pueden ser justificables. (Rodríguez Alva et al., 2016, p. 51)

La validez de un instrumento consiste en que efectivamente se mida el atributo o dimensión para lo cual fue diseñado, enfocándose a que las preguntas que fueron diseñadas para medir una dimensión en particular realmente ayuden a medir lo que se pretende de la variable analizada. Covacevich (2014) citado en Tarazona (2020) define que "La validez se refiere a si el instrumento mide lo que sus autores establecen"(p. 181).

La validez se revisa por medio de un análisis factorial con el cual se busca "descubrir la estructura subyacente de un conjunto de datos cuantitativos definiendo un pequeño número de dimensiones latentes comunes que expliquen la mayor parte de la varianza observada en un conjunto más amplio de variables." (López-Aguado et al., 2019, p. 3) Sin embargo, antes de realizar este análisis 
deben aplicarse las pruebas de esfericidad de Bartlett y Kaiser-Meyer Olkin (KMO). La esfericidad de Barttlet busca demostrar que no están correlacionadas en la muestra las variables que se analizan y la KMO nos demuestra el grado en que las variables se influyen unas a otras. (López-Aguado et al., 2019, pp. 6-7) Los valores aceptables de la prueba KMO entre 0.5 y 1 y la esfericidad de Bartlett debe mostrar valores de significancia menor a 0.05 para considerarse dentro de los parámetros para efectuar análisis posteriores (Crombet-Grillet et al., 2019).

\section{MÉTODO}

La empresa TDV dedicada a la elaboración de Tarimas en Mexicali, Baja California, México, presenta uno de los problemas más comunes de las PYMEs, el cual trata sobre la rotación de personal, por tal motivo este estudio pretende medir la influencia que tienen las variables de sueldos y liderazgo en el índice de rotación de personal en este tipo de empresas. Esta investigación se llevará a cabo mediante un estudio de tipo descriptivo y diseño mixto, ya que se soportará en encuestas de salida, entrevistas de ambiente organizacional a aquellos trabajadores que aún forman parte de la organización, así como revisión documental del historial de estos datos durante el periodo que lleva la administración actual y en el que se han implementado diferentes estadísticas o toma de datos. El inicio de la investigación parte de la literatura relacionada con temas de administración, tesis, artículos científicos y el análisis de los índices de rotación con los que cuenta la empresa, así como datos generados por las cámaras empresariales de la localidad, para con ello poder determinar los factores de mayor incidencia en este fenómeno.

En base a la literatura consultada se determinaron las variables con sus dimensiones, las cuales permiten establecer el impacto que tienen el liderazgo y los sueldos competitivos en la decisión que toman los trabajadores para terminar su relación laboral con la empresa estudiada. Al realizar la operacionalización de las variables (ver tabla 1) se obtuvo un instrumento de medición tipo encuesta que se conforma con 10 preguntas generales con respuestas abiertas y 57 preguntas de escala Likert, dando la posibilidad al sujeto de estudio de cinco opciones para sus respuestas, mismas que se validaran mediante una prueba piloto aplicada a veinte trabajadores del área de producción, de una población total de 75 colaboradores pertenecientes a las celdas (áreas productivas) con un mayor índice de rotación.

Tabla 1. Operacionalización de las Variables

\begin{tabular}{clcc}
\hline Variable & \multicolumn{1}{c}{ Dimensión } & Preguntas & Items \\
\hline \multicolumn{2}{c}{ Preguntas de Identificación general } & & 10 \\
& Selección & S1-S8 & 8 \\
Rotación de Personal & Capacitación & C9-C15 & 7 \\
& Condiciones Laborales & CL16-CL24 & 9 \\
\multirow{3}{*}{ Salario } & Parte Económica & EC25-EC-33 & 9 \\
& Parte Emocional & EM34-EM41 & 8 \\
\multirow{2}{*}{ Liderazgo } & Manejo de personal & MP42-MP49 & 8 \\
& Motivación & MT50-MT57 & 8 \\
\hline & \multicolumn{2}{c}{ Elaboración propia del autor }
\end{tabular}

Los resultados de la prueba piloto se capturaron para su análisis en el programa estadístico IBM SPSS, aplicando para verificar la fiabilidad del instrumento el Alpha de Cronbach en donde se buscan valores mayores que 0.7 y lo más cercanos a 0.9 (Tarazona Mirabal, 2020), aquellos ítems que se encuentran en estos valores deben considerarse en el instrumento final. Para el análisis de validez del instrumento, se aplicaron las pruebas de Esfericidad de Bartlett y KMO (Kaiser-Meyer-Olkin) cuyos valores deben ser menor a 0.05 y superior a 0.5 lo más cercano a 1, respectivamente. 
(Crombet-Grillet et al., 2019) y por ultimo se calcularon las comunalidades que como proponen Hair et al. (1998/1999) "que las variables con una comunalidad menor a 0.5 carecen de una explicación suficiente y no deberían ser consideradas en la interpretación final del análisis." (Zamora Muñoz et al., 2010, p. 27), por lo que aquellos ítems con un valor menor a 0.40 no se tomaron en cuenta en este análisis.

Después de la aplicación de las pruebas mencionadas al instrumento diseñado, se conservan 38 preguntas que muestran los valores adecuados para conservarse en el instrumento final a aplicar para determinar las variables que más influyen en la rotación laboral de la empresa TDV.

\section{RESULTADOS.}

La encuesta fue validada por una variable dependiente que es la rotación de personal e integrada por 13 preguntas, así como por dos variables independientes salario y liderazgo, demostrando la manera en que influyen en la rotación de personal. La variable de Salario consta de 12 preguntas en dos dimensiones, parte económica y parte emocional. El liderazgo fue evaluado por medio de 13 preguntas divididas en dos dimensiones el manejo de personal y la motivación. (Ver tabla 1.)

Como se puede observar en la Tabla 2. las dimensiones correspondientes a Rotación de Personal muestran en la prueba de fiabilidad Alpha de Cronbach valores dentro de los parámetros aceptables, por lo que el instrumento es fiable para estas dimensiones. Al aplicar las pruebas de validez KMO y Bartlett a dichas dimensiones, se obtienen resultados dentro de los parámetros admisibles por lo tanto se considera que el instrumento es válido.

Las pruebas realizadas al instrumento fueron Alpha de Cronbach, KMO y Bartlett, donde Selección de personal obtuvo; 0.746; 0.564; 0.002, Capacitación; 0.944; 0.828; 0.000 y por último Condiciones laborales con; $0.917 ; 0.634 ; 0.000$ respectivamente, lo que significa que las pruebas de validez $y$ fiabilidad fueron debidamente cubiertas por los parámetros establecidos como aceptables.

Tabla 2. Alpha de Cronbach, KMO, Bartlett de variable dependiente

\begin{tabular}{|c|c|c|c|c|c|c|}
\hline \multirow{3}{*}{ Variable } & \multirow{3}{*}{ Dimensión } & \multirow{3}{*}{ Preguntas } & \multirow{3}{*}{ Items } & \multicolumn{3}{|c|}{ Pruebas realizadas } \\
\hline & & & & $0.7-0.9$ & $0.5-1$ & $<.05$ \\
\hline & & & & $\begin{array}{l}\text { Alpha de } \\
\text { Cronbach }\end{array}$ & KMO & BARTLETT \\
\hline \multirow{3}{*}{$\begin{array}{c}\text { Rotación de } \\
\text { Personal }\end{array}$} & Selección & S1-S4 & 4 & 0.746 & 0.564 & 0.002 \\
\hline & Capacitación & C5-C10 & 6 & 0.944 & 0.828 & 0.000 \\
\hline & Condiciones Laborales & CL11-CL13 & 3 & 0.917 & 0.634 & 0.000 \\
\hline
\end{tabular}

La variable de Salario y su dimensión parte económica que consta de 6 preguntas, obtuvo en la prueba de Alpha de Cronbach un valor de 0.902, un KMO 0.653 y Bartlett 0.000 lo que indica que se encuentran dentro de los valores adecuados. (Ver tabla 3.)

Tabla 3. Alpha de Cronbach, KMO, Bartlett de Salario (parte económica)

\begin{tabular}{|c|c|c|c|c|c|c|}
\hline \multirow{3}{*}{ Variable } & \multirow{3}{*}{ Dimensión } & \multirow{3}{*}{ Preguntas } & \multirow{3}{*}{ Items } & \multicolumn{3}{|c|}{ Pruebas realizadas } \\
\hline & & & & $0.7-0.9$ & $0.5-1$ & $<.05$ \\
\hline & & & & $\begin{array}{l}\text { Alpha de } \\
\text { Cronbach }\end{array}$ & KMO & BARTLETT \\
\hline Salario & Parte Económica & EC14-EC19 & 6 & 0.902 & 0.653 & 0.000 \\
\hline
\end{tabular}


La dimensión del salario, parte emocional dividida en 6 preguntas, generó un Alpha de Cronbach con un valor de 0.863 así como un KMO 0.789 y una esfericidad de
Bartlett con 0.003, valores que indican que se encuentran dentro de los parámetros. (Ver tabla 4.

Tabla 4. Alpha de Cronbach, KMO, Bartlett de Salario (parte emocional)

\begin{tabular}{|c|c|c|c|c|c|c|}
\hline \multirow{4}{*}{ Variable } & \multirow{3}{*}{ Dimensión } & \multirow{3}{*}{ Preguntas } & \multirow{3}{*}{ Items } & \multicolumn{3}{|c|}{ Pruebas realizadas } \\
\hline & & & & $0.7-0.9$ & $0.5-1$ & $<.05$ \\
\hline & & & & $\begin{array}{l}\text { Alpha de } \\
\text { Cronbach }\end{array}$ & KMO & BARTLETT \\
\hline & Parte Emocional & EM20-EM25 & 6 & 0.863 & 0.789 & 0.003 \\
\hline
\end{tabular}

La siguiente dimensión analizada corresponde a la variable de Liderazgo, enfocada a la forma en que el líder de las celdas productivas cuenta con un adecuado manejo de personal a su cargo, dicha dimensión se divide en cinco preguntas. Se aplicó la prueba del Alpha de Cronbach para verificar la fiabilidad del instrumento en esta dimensión obteniendo un valor de 0.947 , las pruebas de KMO y esfericidad de Bartlett mostraron valores dentro de los parámetros idóneos de 0.629 y 0.000 respectivamente. (ver tabla 5.)

Tabla 5. Alpha de Cronbach, KMO, Bartlett de Liderazgo (Manejo de personal)

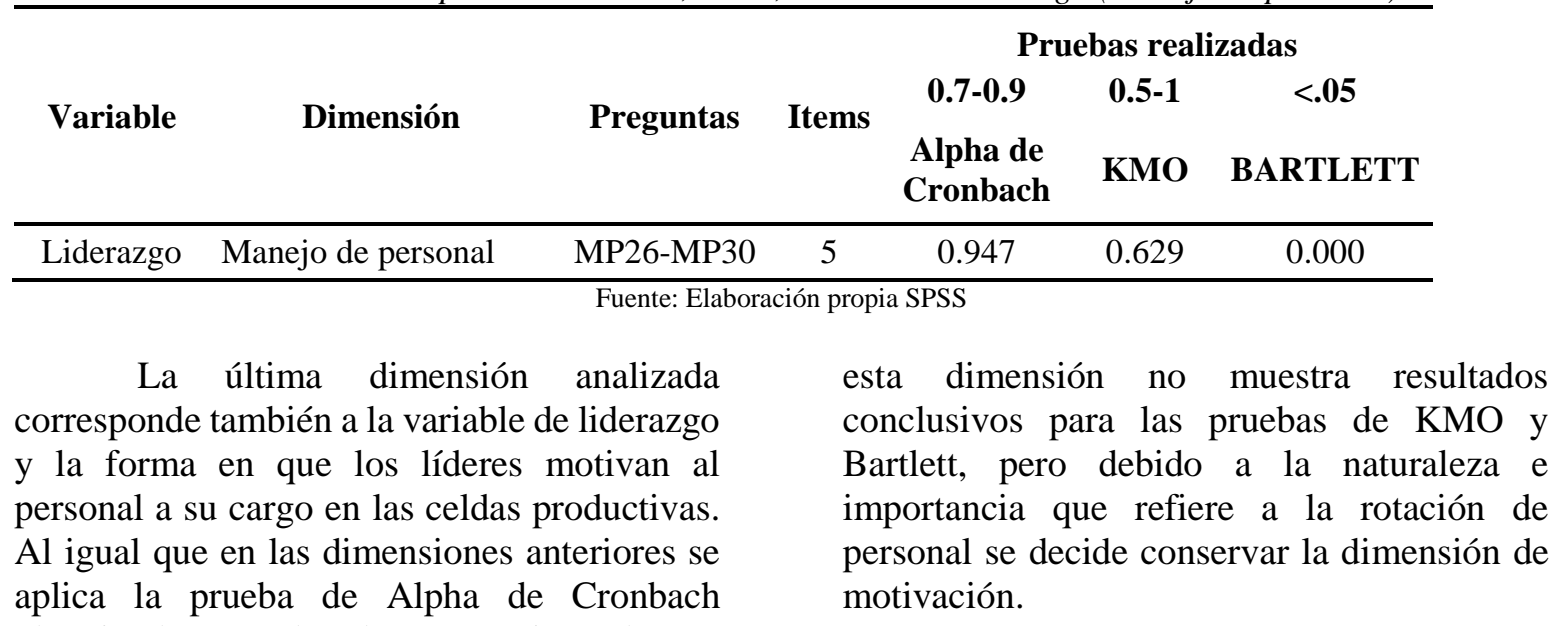
obteniendo un valor de 0.960 , sin embargo,

Tabla 6. Alpha de Cronbach, KMO, Bartlett de Liderazgo (Motivación)

\begin{tabular}{|c|c|c|c|c|c|c|}
\hline \multirow{3}{*}{ Variable } & \multirow{3}{*}{ Dimensión } & \multirow{3}{*}{ Preguntas } & \multirow{3}{*}{ Items } & \multicolumn{3}{|c|}{ Pruebas realizadas } \\
\hline & & & & $0.7-0.9$ & $0.5-1$ & $<.05$ \\
\hline & & & & $\begin{array}{l}\text { Alpha de } \\
\text { Cronbach }\end{array}$ & KMO & BARTLETT \\
\hline Liderazgo & Manejo de personal & MT31-MT38 & 8 & 0.960 & & \\
\hline
\end{tabular}

A continuación, se presentan las tablas de comunalidades de cada uno de los factores, donde se puede observar que son "aceptablemente explicadas por el modelo" 
(Zamora Muñoz et al., 2010, p. 27) debido a que se muestran valores por encima de .40 (ver tabla 7.)

Tabla 7. Comunalidades

\begin{tabular}{|c|c|c|c|c|c|}
\hline Items & Inicial & Extracción & Items & Inicial & Extracción \\
\hline S1 & 1.000 & .625 & EM20 & 1.000 & .628 \\
\hline S2 & 1.000 & .734 & EM21 & 1.000 & .721 \\
\hline S3 & 1.000 & .454 & EM22 & 1.000 & .651 \\
\hline S4 & 1.000 & .538 & EM23 & 1.000 & .582 \\
\hline C5 & 1.000 & .844 & EM24 & 1.000 & .655 \\
\hline C6 & 1.000 & .759 & EM25 & 1.000 & .532 \\
\hline C7 & 1.000 & .829 & MP26 & 1.000 & .657 \\
\hline $\mathrm{C} 8$ & 1.000 & .891 & MP27 & 1.000 & .799 \\
\hline C9 & 1.000 & .750 & MP28 & 1.000 & .961 \\
\hline $\mathrm{C} 10$ & 1.000 & .687 & MP29 & 1.000 & .857 \\
\hline CL11 & 1.000 & .941 & MP30 & 1.000 & .876 \\
\hline CL12 & 1.000 & .855 & MT31 & 1.000 & .678 \\
\hline CL13 & 1.000 & .781 & MT32 & 1.000 & .807 \\
\hline EC14 & 1.000 & .786 & MT33 & 1.000 & .857 \\
\hline EC15 & 1.000 & .771 & MT34 & 1.000 & .818 \\
\hline EC16 & 1.000 & .711 & MT35 & 1.000 & .798 \\
\hline EC17 & 1.000 & .698 & MT36 & 1.000 & .857 \\
\hline EC18 & 1.000 & .619 & MT37 & 1.000 & .811 \\
\hline EC19 & 1.000 & .639 & MT38 & 1.000 & .780 \\
\hline
\end{tabular}

Posteriormente a las pruebas realizadas y debido a los resultados obtenidos se determinó eliminar 19 preguntas que afectaban los rangos de fiabilidad y validez del instrumento, quedando el instrumento final con 38 preguntas, mismo que se puede observar en los anexos. (Ver anexo 1)

\section{CONCLUSIONES}

Una vez realizadas las pruebas estadísticas de fiabilidad y validez al instrumento diseñado para medir la rotación de personal, se puede concluir se cuenta con instrumento fiable y valido, debido a que los valores resultantes se encuentran dentro de los parámetros adecuados conforme a los autores revisados en la literartura.

Los sujetos encuestados en la realización de la prueba piloto fueron cooperativos y debido a sus aportaciones en el sentido de claridad de la redacción de las preguntas, se pudo identificar aquellas palabras que ocasionaron confusión, mismas que fueron resideñadas de acuerdo a sus comentarios para una mejor comprensión.

Al contar con un instrumento ya validado que indica, de acuerdo a las pruebas a las que se sometió y sus resultados, que mediante éste se mediarán de manera adecuada la influencia de las varibles independientes (Salarios y liderazgo) sobre la variable dependiente (rotación laboral). Lo que permitirá continuar con la aplicación de la encuesta final al total de la población elegida para obtener los resultados para lo cual fue diseñado éste instrumento.

La rotación laboral es un fenómeno que aqueja actualmente a diversas empresas y que se ha estudiado de diferentes ángulos, debido a los altos costos que se genera en las 
mismas, siendo no sólo económicos, ya que también se ve impactado el ambiente laboral en que se desarrollan las actividades diarias de los sectores productivos.

De igual manera, se observa que el impacto de dicho fenómeno no se dá solamente en las áreas productivas que presentan un mayor índice de rotación, sino también en todos aquellos departamentos administrativos encargados del control de nómina, contratación, selección y capacitación de los colaboradores de la empresa.

El instrumento validado que se presenta, será de utilidad a la empresa TDV y Pymes con carcaterísticas similares en cuanto a actividad, ubicación y tipo de planta laboral, permitiéndoles analizar el impacto que tienen en la rotación laboral los factores de salarios y liderazgo, con el fin de diseñar y utilizar las estrategias adecuadas conforme a los resultados que se obtendrán al aplicar éste instrumento a su plantilla de trabajo.
Al momento de aplicar la encuesta piloto, el tiempo que se dió a los participantes para las respuestas fue corto debido a que la empresa se encuentra en temporada alta, por lo que se tuvo esta limitación en el desarrollo del trabajo, por lo cual se recomienda analizar estos tiempos con los encargados, al momento de la aplicación del instrumento al total de los colaboradores de la empresa.

Los resultados del trabajo aquí propuesto, pueden ser de interés para las empresas que se encuentran en el área del Valle de Mexicali, ya que las características del personal contratado no son las mismas que se manejan en las zonas urbanas cercanas, dándose las condiciones para que a futuro, se permita analizar a una mayor población y conformada por personal de diversas empresas. 


\section{REFERENCIAS}

Aldape, A., Pedrozo, J., Castillo Pérez, V. H., \& Moguel Liévano, M. de J. (2011). Liderazgo y satisfacción en el trabajo vale la pena estudiarlos. Revista de Economía, Finanzas y Negocios, 2(1). https://www.researchgate.net/publication/281595352

Aranibar Gutiérrez, M. F., Melendres Carlos, V. D., Ramírez Barón, M. C., \& García Rivera, B. R. (2018). Los factores de la rotación de personal en las maquiladoras de exportación de Ensenada, B.C. Revista global de negocios, 6(2), 22-25.

Barón Ramírez, A. (2020). Incidencia de uso de las prestaciones laborales superiores en la rotación de personal de una empresa maquiladora de la ciudad de Ensenada. Un estudio de caso. [Maestría]. Universidad Autónoma de Baja California.

Benavides Coronado, E. M., \& Pimentel Felix, A. F. (s/f). Liderazgo conductual y causas externas e internas de rotación de los operadores. http://www.semac.org.mx/archivos/5-14.pdf

Canu, M., \& Duque, M. (2017). SOBRE EL COEFICIENTE ALPHA DE CRONBACH Y SU INTERPRETACIÓN EN LA EVALUACIÓN EDUCATIVA. Encuentro Internacional de Educación en Ingeniería. https://acofipapers.org/index.php/eiei/article/view/608/612

Chiavenato, I. (2009). Gestión del talento humano, Tercera Edición (Tercera). McGrawHill/Interamericana Editores, S.A. de C.V.

Chiavenato, I. (2011). Administración de Recursos Humanos: El capital humano de las organizaciones, Novena Edición (Novena). McGraw-Hill/Interamericana Editores, S.A. de C.V.

Cifuentes Cadena, T. (2017). Análisis de factores que influyen en la rotación de personal en una empresa del rubro de alimentos y bebidas [Magister, Univeresidad de Chile]. http://repositorio.uchile.cl/bitstream/handle/2250/149838/Cifuentes\%20Cadena\%20Tatiana .pdf?sequence $=1 \&$ isAllowed $=y$

Crombet-Grillet, S., Abalos-Rodríguez, A., Zamora-Matamoros, L., Crombet-Grillet, S., AbalosRodríguez, A., \& Zamora-Matamoros, L. (2019). Determinación de los parámetros ambientales de mayor incidencia en las aguas residuales de la comunidad universitaria Antonio Maceo. Revista Cubana de Química, 31(1), 137-153.

Domínguez Olaya, M. K. D. (2015). Análisis de las causas de rotación de personal de la empresa Holcrest S.A.S [Maestría]. Universidad de Medellín.

Flores, R., Abreu, J. L., \& Badii, M. H. (2008). Factores que originan la rotación de personal en las empresas mexicanas. Revista Daena (International Journal of Good Conscience, 3(1), 6591.

García Rivera, B. R., Ramírez Barón, M. C., \& Aranibar Gutiérrez, M. F. A. (2020). La rotación de personal del empleado directo: La satisfacción y el compromiso como mediadores. Revista de Desarrollo Sustentable, Negocios, Emprendimiento y Educación., 6.

Gibson, J. L., Ivancevich, J. M., Donelly, Jr, J. H., \& Konopaske, R. (2011). Organizaciones: Comportamiento, estructura y procesos (Decimotercera). McGraw-Hill/Interamericana Editores, S.A. de C.V. https://libcon.rec.uabc.mx:4431

Herrera Arau, K. V. (2018). Estabilidad laboral y su relación con la satisfacción, compromiso organizacional y flexibilidad en empresa textil de Ensenada, Baja California [Maestría]. Universidad Autónoma de Baja California.

Koontz, H., \& Weihrich, H. (2013). Elementos de administración: Un enfoque internacional y de innovación. (8a.). McGraw-Hill/Interamericana Editores, S.A. de C.V. https://libcon.rec.uabc.mx:4431/lib/uabcsp/detail.action?docID=3214420

Ley Federal del Trabajo, (2019).

Llanes Barreras, E. (2019). Propuesta de programa integral para minimizar la rotación del personal en empresas de ventas al detalle en Mexicali, B.C. [Maestría]. Universidad Autónoma de Baja California. 
López-Aguado, M., \& Gutiérrez-Provecho, L. (2019). Cómo realizar e interpretar un análisis factorial exploratorio utilizando SPSS. REIRE Revista d'Innovació i Recerca en Educació, $12(2), 1-14$.

Newstrom, J. W. (2011). Comportamiento humano en el trabajo (Decimotercera). McGrawHill/Interamericana Editores, S.A. de C.V.

Patista Serrano, D. M., \& Reyes García, J. I. (2019). Factores que inciden en la rotación de personal en las mipymes afiliadas a la Canacintra. XXIV Congreso internacional de contaduría, administración e informática., Ciudad de México. http://132.248.164.227/congreso/docs/xxiv/docs/1.03.pdf

Ramos García, J. R. (2018). Análisis de las dimensiones del clima organizacional que inciden en la rotación de personal en la empresa Splash Car Wash de Mexicali, B.C. [Maestría]. Universidad Autónoma de Baja California.

Robbins, S. P. (2009). Comportamiento organizacional. Pearson Educación.

Rodríguez Alva, J. P., Ortiz Puente de la Vega, J. H., Vera Zavala, G. E., Soto Carpio, J. J., \& Delgado Palomino, J. A. (2016). Satisfacción y Rotación Laboral en Personal de Empresas Mineras de Arequipa y Cusco [Maestría, UNIVERSIDAD CATÓLICA DEL PERÚ]. http://hdl.handle.net/20.500.12404/8638

Tarazona Mirabal, H. (2020). Observaciones para la construcción y validación de instrumentos de investigación. Desafios, 11(2), 70-83.

Vázquez, X. (2006). Rotación De Personal En La Maquiladora: ¿La Satisfacción Laboral, Liderazgo Y Perfil Sociodemográfico, Importan? Gestiopolis. https://www.gestiopolis.com/wp-content/uploads/2008/07/rotacion-de-personal-dentro-delos-recursos-humanos.pdf

Zamora Muñoz, S., Monroy Carzola, L., \& Chávez Álvarez, C. (2010). Análisis factorial: Una técnica para evaluar la dimensionalidad de las pruebas Cuaderno técnico 6 [Review of Análisis factorial: Una técnica para evaluar la dimensionalidad de las pruebas Cuaderno técnico 6, por A. Saade Jazin]. Centro Nacional de Evaluación para la Educación Superior, A.C. (Ceneval).

https://www.ceneval.edu.mx/documents/20182/0/CuadernoTecnico061aed.pdf/a922a29593f3-4bd0-9c8b-28a52c499cf5 


\section{Anexos}

Anexo 1. Instrumento Final

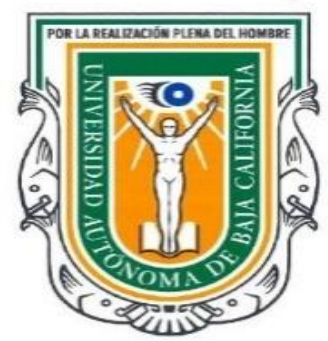

\section{UNIVERSIDAD AUTÓNOMA DE BAJA CALIFORNIA}

Facultad de Ciencias Administrativas

Caso de Estudio: Variables que afectan la rotación laboral en TDV.

Esta entrevista es realizada con fines de investigación académica, se busca obtener información sobre temas relacionados con la empresa en donde labora, los datos proporcionados serán manejados de forma confidencial y anónima.

\section{A. INFORMACIÓN GENERAL}

Favor de llenar los espacios con la información solicitada.

Edad

Género Masculino Femenino No especifica

Estado Civil

Tiempo trabajando en la empresa

Tiempo en el puesto actual
Escolaridad

Puesto desempeñado

No. de hijos

Celda

Productiva

\section{Lugar de residencia}

\section{B. DESARROLLO DE LA ENCUESTA}

Favor marcar con una " X" la respuesta que considere más adecuada.

\section{Selección}

Considero que los términos de

1 contratación son de acuerdo con mis expectativas.

2 El salario ofrecido cumple con mis expectativas.

3 La empresa es mi mejor opción para trabajar.

4 La empresa ofrece buenas prestaciones.

\begin{tabular}{|l|l|l|l|l|}
\hline $\begin{array}{c}\text { Totalmente } \\
\text { de acuerdo }\end{array}$ & $\begin{array}{c}\text { De } \\
\text { acuerdo }\end{array}$ & Indiferente & $\begin{array}{c}\text { En } \\
\text { desacuerdo }\end{array}$ & $\begin{array}{c}\text { Totalmente } \\
\text { en } \\
\text { desacuerdo }\end{array}$ \\
\hline & & & & \\
\hline & & & & \\
\hline & & & & \\
\hline & & & & \\
\hline
\end{tabular}


Capacitación

5 Continuamente recibo cursos de capacitación por parte de la empresa.

6

La capacitación que recibo es adecuada para desempeñar mi trabajo.

Se me capacita continuamente para

7 desempeñar diferentes puestos dentro de la empresa.

Considero que, con la capacitación

8 recibida, mejoro mis oportunidades de desarrollo en la empresa.

9 Puedo hacer mejor mi trabajo debido a las capacitaciones recibidas.

En el tiempo que ha estado en la empresa,

10 he asistido a dos o más cursos de capacitación.

\section{Condiciones Laborales}

11

La empresa me otorga el equipo adecuado para realizar mi trabajo.

12

El espacio de trabajo es adecuado para desarrollar mis actividades laborales.

13

Las condiciones de trabajo son adecuadas para continuar laborando en la empresa.

Remuneraciones (aspecto económico)

14

Los bonos de producción se otorgan de forma equitativa para todo el personal.

El bono de puntualidad me motiva a llegar en tiempo.

El bono de asistencia me motiva a no faltar al trabajo.

17

Los bonos de producción son equitativos con los estándares solicitados.

18

Estoy satisfecho con el ingreso que gano semanalmente.

19

El bono de antigüedad me motiva a permanecer en la empresa.

\begin{tabular}{|l|l|l|l|l|}
\hline $\begin{array}{c}\text { Totalmente } \\
\text { de acuerdo }\end{array}$ & $\begin{array}{c}\text { De } \\
\text { acuerdo }\end{array}$ & Indiferente & $\begin{array}{c}\text { En } \\
\text { desacuerdo }\end{array}$ & $\begin{array}{c}\text { Totalmente } \\
\text { en } \\
\text { desacuerdo }\end{array}$ \\
\hline & & & & \\
\hline & & & & \\
\hline & & & & \\
\hline & & & & \\
\hline & & & & \\
\hline
\end{tabular}

\begin{tabular}{|l|l|l|l|l|}
\hline $\begin{array}{c}\text { Totalmente } \\
\text { de acuerdo }\end{array}$ & $\begin{array}{c}\text { De } \\
\text { acuerdo }\end{array}$ & Indiferente & $\begin{array}{c}\text { En } \\
\text { desacuerdo }\end{array}$ & $\begin{array}{c}\text { Totalmente } \\
\text { en } \\
\text { desacuerdo }\end{array}$ \\
\hline & & & & \\
\hline & & & & \\
\hline & & & & \\
\hline
\end{tabular}

\begin{tabular}{|l|l|l|l|l|}
\hline $\begin{array}{c}\text { Totalmente } \\
\text { de acuerdo }\end{array}$ & $\begin{array}{c}\text { De } \\
\text { acuerdo }\end{array}$ & Indiferente & $\begin{array}{c}\text { En } \\
\text { desacuerdo }\end{array}$ & $\begin{array}{c}\text { Totalmente } \\
\text { en } \\
\text { desacuerdo }\end{array}$ \\
\hline & & & & \\
\hline & & & & \\
\hline & & & & \\
\hline & & & & \\
\hline & & & & \\
\hline
\end{tabular}


Remuneraciones (aspecto emocional)

\begin{tabular}{|l|l|l|l|l|}
\hline $\begin{array}{c}\text { Totalmente } \\
\text { de acuerdo }\end{array}$ & $\begin{array}{c}\text { De } \\
\text { acuerdo }\end{array}$ & Indiferente & $\begin{array}{c}\text { En } \\
\text { desacuerdo }\end{array}$ & $\begin{array}{c}\text { Totalmente } \\
\text { en } \\
\text { desacuerdo }\end{array}$ \\
\hline & & & & \\
\hline & & & & \\
\hline & & & & \\
\hline & & & & \\
\hline
\end{tabular}

\section{Liderazgo (manejo de personal)}

Recibo la orientación adecuada por parte

26 de mi supervisor para desempeñar adecuadamente mi trabajo.

Mi supervisor genera confianza en el equipo para resolver situaciones laborales. Mi jefe me ayuda a resolver dudas o

28 problemas que afectan mi ingreso semanal.

El diálogo entre mi supervisor y el equipo de trabajo es fluido y respetuoso.

Recibo información clara de mi supervisor para desempeñar mis labores.

\begin{tabular}{|l|l|l|l|l|}
\hline $\begin{array}{c}\text { Totalmente } \\
\text { de acuerdo }\end{array}$ & $\begin{array}{c}\text { De } \\
\text { acuerdo }\end{array}$ & Indiferente & $\begin{array}{c}\text { En } \\
\text { desacuerdo }\end{array}$ & $\begin{array}{c}\text { Totalmente } \\
\text { en } \\
\text { desacuerdo }\end{array}$ \\
\hline & & & & \\
\hline & & & & \\
\hline & & & & \\
\hline & & & & \\
\hline & & & & \\
\hline
\end{tabular}


Liderazgo (motivación)

31 Mi supervisor toma en cuenta mis opiniones para el desarrollo del trabajo.

32 Mi desempeño en el trabajo es reconocido por mi líder.

33 Mi líder presta atención al trabajo que realizo.

34 Mi bienestar en el desempeño de mi trabajo es importante para mi jefe.

Mi líder me motiva a mejorar en mi trabajo.

36 Me siento a gusto trabajando con mi líder.

37 Mis superiores consideran que mis labores desempeñadas son dignas de respeto.

Mis superiores están al pendiente de mis

38 necesidades para el adecuado desempeño de mi trabajo

\begin{tabular}{|l|l|l|l|l|}
\hline $\begin{array}{c}\text { Totalmente } \\
\text { de acuerdo }\end{array}$ & $\begin{array}{c}\text { De } \\
\text { acuerdo }\end{array}$ & Indiferente & $\begin{array}{c}\text { En } \\
\text { desacuerdo }\end{array}$ & $\begin{array}{c}\text { Totalmente } \\
\text { en } \\
\text { desacuerdo }\end{array}$ \\
\hline & & & & \\
\hline & & & & \\
\hline & & & & \\
\hline & & & & \\
\hline & & & & \\
\hline
\end{tabular}

MUCHAS GRACIAS POR SUS RESPUESTAS. 